\title{
A PROPERTY OF THE PERIODS OF A PRYM DIFFERENTIAL
}

\author{
GEORGE KEMPF 1
}

\begin{abstract}
The periods of Prym differentials can be used to prove the invariance of Picard bundles on Jacobian varieties.
\end{abstract}

Let $S$ be a compact Riemann surface of genus $g$ with universal covering surface $U$ with the deck transformation group $D$. For any homomorphism $X: D \rightarrow \mathbf{C}^{*}=\mathbf{C}-\{0\}$ into the group of complex units, a (meromorphic) Prym differential on $S$ with multipliers $X$ is a meromorphic differential $w$ on $U$, satisfying the condition

$$
w(d u)=X(d) w(u) \text { for all } d \text { in } D \text { and } u \text { in } U .
$$

Let $p$ be a fixed point of $U$. For all $X$, consider the space of Prym differentials which are regular except for a pole of at most order one at $p$ or its translates under $D$. This space has dimension $g$ and varies complex analytically as one varies $X$ (see below). These spaces form a vector bundle over the space of all $X$. In this paper, I show how to trivialize this vector bundle over a $g$ dimensional real torus, consisting of special $X$ 's, by means of the periods of Prym differentials.

In a previous paper [3], I proved that such a trivialization was possible by abstract reasoning. The methods of this paper are more elementary and yield an explicit trivialization. Some basic facts about this vector bundle can be found in [2]. A general reference for periods of Prym differentials is the book [1].

Given any Prym differential $w$, let $V$ be the largest open subset of $U$ such that $w$ has zero residues everywhere in $V$. Denote the image of $V$ in $S$ by $T$.

Let $v$ be any fixed point of $V$ and let $t$ be its image in $T$. For any path $\sigma$ in $T$, beginning and ending at $t$, the $\sigma$ period $A_{\sigma}$ of $w$ is defined by the integral

$$
A_{\sigma}=-X(\underline{\sigma})^{-1} \int_{\tilde{\sigma}} w,
$$

where $\tilde{\sigma}$ is the unique path in $U$, lifting $\sigma$, which begins at $t$ and ends at $\underline{\sigma} \cdot t$. The deck transformation $\sigma$ will henceforth be denoted by $\sigma$. Thus, $A_{\sigma}$ depends only on the homotopy class of $\sigma$ in the fundamental group of $T$. Furthermore, $A_{\sigma}$ is defined for most $\sigma$ in any homotopy class. We can then regard $A_{\sigma}$ as a function on $\pi_{1}(T, t)$. Also, it satisfies the cocycle identity,

$$
A_{\sigma \tau}=X(\tau)^{-1} A_{\sigma}+A_{\tau} \text { for all } \sigma \text { and } \tau \text { in } \pi_{1}(T, t) .
$$

Received by the editors February 5, 1975.

AMS (MOS) subject classifications (1970). Primary 32L05, 14K30.

Key words and phrases. Prym differential, Jacobian variety, vector bundle.

1 Partly supported by the Sloan Foundation. 
Let $[\sigma, \tau]$ be the commutator $\sigma \tau \sigma^{-1} \tau^{-1}$. The cocycle identity implies that

$$
A_{[\sigma, \tau]}=X(\tau) X(\sigma)\left[\left(1-X(\sigma)^{-1}\right) A_{\tau}-\left(1-X(\tau)^{-1}\right) A_{\sigma}\right] .
$$

Let $\sigma_{1}, \tau_{1}, \ldots, \sigma_{g}, \tau_{g}$ be a standard set [1] of closed arcs, beginning and ending at $t$, that dissect $S$. Let $R$ be the area in $U$ bounded by the lifting $\tau_{\left[\tau_{1}, \sigma_{1}\right] \cdots\left[\tau_{g}, \sigma_{g}\right]}$.

Proposition 1. Let $w$ be a Prym differential with multipliers $X$. If $w$ has no poles on the boundary of $R$, then

$$
-2 \pi i\left(\sum_{u \in R} \text { Residue of } w \text { at } u\right)=\sum_{1 \leqq i \leqq g} A_{\left[\tau_{i}, \sigma_{i}\right]} \text {. }
$$

The proof of this proposition is a straightforward application of the residue formula and the cocycle identity.

The other fact that I will need is

Proposition 2. Let $w_{1}$ be a Prym differential with multipliers $X$ and periods $A$, and let $w_{2}$ be a Prym differential with conjugate inverse multipliers $\bar{X}^{-1}$ and periods $B$. Let $w_{1}$ and $w_{2}$ have no poles on the boundary of $R$. Assume that

(1) $w_{1}$ has zero residues everywhere, and

(2) order $w_{1}+$ order $w_{2} \geqq-1$ everywhere. Then $\int_{R} \bar{w}_{2} \wedge w_{1}$ equals the sum

$$
\begin{aligned}
\sum_{1 \leqq j \leqq g} & \bar{B}_{\tau_{j}} X\left(\tau_{j}\right)^{-1} A_{\sigma_{j}}-\bar{B}_{\sigma_{j}} X\left(\sigma_{j}\right)^{-1} A_{\tau_{j}} \\
& \quad-\bar{B}_{\sigma_{j}} X\left(\tau_{j} \sigma_{j}\right)^{-1}\left(A_{\lambda_{j-1}}-X\left(\tau_{j}\right) A_{\lambda_{j}}\right)+\bar{B}_{\tau_{j}} X\left(\sigma_{j} \tau_{j}\right)^{-1}\left(A_{\lambda_{j}}-X\left(\sigma_{j}\right) A_{\lambda_{j-1}}\right)
\end{aligned}
$$

where $\lambda_{j}=\Pi_{1 \leqq i \leqq j}\left[\tau_{i}, \sigma_{i}\right]$.

Proof. The second assumption assures that the integral $\int_{R} \bar{w}_{2} \wedge w_{1}$ converges. By the first assumption, we can find a meromorphic function $F$ on $U$ such that $d F=w_{1}$ and $F(v)=0$. Then $F(d u)=X(d)\left(F(u)-A_{d}\right)$ for all $d$ in $D$ and all $u$ in $U$. By the calculation in the proof of Theorem 21 in [1], $\int_{R} \bar{w}_{2} \wedge w_{1}=-\int_{\tilde{\lambda}_{g}} w_{2} F$ equals the above expression. Q.E.D.

Let $p$ be any fixed point in the interior of $R$. From the previous two propositions, we can conclude

Proposition 3. Let $w$ be a Prym differential with multipliers $X$. Assume $w$ is regular except for a pole of at most order one at $p$ and its translates. Assume that $|X(d)|=1$ for all $d$ in $D$. Further, assume that $X\left(\sigma_{i}\right)=1$ for all $1 \leqq i \leqq g$. Then $w$ is determined by its $g$ periods $A_{\sigma_{i}}$.

Proof. As $X\left(\sigma_{i}\right)=1$, from equation $(*)$, we get $A_{\left[\tau_{i}, \sigma_{i}\right]}=\left[X\left(\tau_{i}\right)-1\right] A_{\sigma_{i}}$.

Assume the above differential $w$ has all its $\sigma_{i}$ periods equal to zero. Then the $A_{\left[\tau_{i}, \sigma_{i}\right]}$ are zero by the above equation. By the first proposition, the residue of 
$w$ at its only pole in $R$ is zero. Hence, $w$ must be regular at $p$ because its pole has at most order one.

As $X=\bar{X}^{-1}$ by assumption, we may apply the second proposition to compute the integral $\int_{R} \bar{w} \wedge w$ for the regular differential $w$. To do this, let us first notice that $A_{\lambda_{j}}=A_{\lambda_{j-1}}+A_{\left[\tau_{j}, \sigma_{j}\right]}$. As we know from before that the $A\left[\tau_{j}, \sigma_{j}\right]$ are zero, we have that the $A_{\lambda_{j}}$ are all zero. Thus, the formula in the last proposition reduces to

$$
\int_{R} \bar{w} \wedge w=\sum_{1 \leqq j \leqq g} \bar{A}_{\tau_{j}} X\left(\tau_{j}\right)^{-1} A_{\sigma_{j}}-\bar{A}_{\sigma_{j}} A_{\tau_{j}}
$$

The second side is zero because the $A_{\sigma_{j}}$ are. Therefore, $\int_{R} \bar{w} \wedge w=0$, but $\bar{w} \wedge w$ is locally a nonnegative multiple of $\overline{d z} \wedge d z=2 i d x \wedge d y$. Hence, we have $\bar{w} \wedge w=0$. Consequently, $w=0$.

The proposition follows from the above because the periods are linear functionals on the differentials. Q.E.D.

As a homomorphism $X$ is determined by its $2 g$ values $X\left(\sigma_{1}\right), \ldots, X\left(\sigma_{g}\right)$, $X\left(\tau_{1}\right), \cdots, X\left(\tau_{g}\right)$, the set of $X$ 's is a complex manifold $M$ isomorphic to $\times_{2 g} \mathbf{C}^{*}$. The sheaf of regular Prym differentials with multipliers $X$ defines a line bundle $\Omega_{S}(X)$ of degree $2 g-2$ on $S$. The line bundles $\Omega_{S}(X)$ depend complex analytically on $X$. A Prym differential with multipliers $X$, which is regular except for a pole of order at most one at $p$ and its translates, can be considered as a section of $\Omega_{S}(X)(q)$, where $q$ is the image of $p$ in $S$. As $\Omega_{S}(X)(q)$ has degree $2 g-1$, its space of sections $W(X, q)$ has dimension $g$ by the RiemannRoch theorem. Furthermore, as $X$ varies, the family $W(X, q)$ forms a complex analytic vector bundle $W(q)$ with base $M$.

A period $A_{\sigma_{i}}$ defines a complex analytic mapping of $W(q)$ to the trivial bundle. If $X$ is one of the systems of multipliers satisfying the conditions of the third proposition, then the $g$ periods $A_{\sigma_{1}}, \ldots, A_{\sigma_{g}}$ map $W(X, q)$ isomorphically onto a vector space $\mathbf{C}^{g}$. As this will consequently happen for all nearby $X$, the next theorem follows.

THEOREM 1. There is a maximal open subset $N$ of $M$, such that

(1) $N$ contains all multipliers $X$ with $|X(d)|=1$ for all $d$ in $D$ and $X\left(\sigma_{i}\right)=1$ for all $1 \leqq i \leqq g$, and

(2) for any $X$ in $N$, there exists a basis $\left(w_{1}(X), \ldots, w_{g}(X)\right)$ of $W(X, q)$ such that the $\sigma_{i}$ period of $w_{j}(X)$ is one if $i$ equals $j$ and is zero otherwise. Furthermore, the $w_{i}(X)$ depend complex analytically on $X$, and form a basis for the vector bundle $W(q)$ restricted to $N$.

As noted in the previous paper [3], a theorem of Grauert and the first theorem imply

THEOREM 2. $W(p)$ is a complex analytically trivial bundle when it is restricted to the manifold of $X$ satisfying $X\left(\sigma_{i}\right)=1$ for $1 \leqq i \leqq g$.

Further questions of interest would be: How can one prove the second theorem directly? How large is the open $N$ in the first theorem? What can you say about the $\tau$ periods of the normalized Prym differentials $w_{i}(X)$ ? 


\section{BIBLIOGRAPHY}

1. R. C. Gunning, Lectures on vector bundles over Riemann surfaces, Univ. of Tokyo Press, Tokyo; Princeton Univ. Press, Princeton, N. J., 1967. MR 37 \# 5888.

2. —- Some special complex vector bundles over Jacobi varieties, Invent. Math. 22 (1973), 187-210.

3. George R. Kempf, Some vector bundles on Jacobian varieties, Proc. Amer. Math. Soc. 54 (1976), 179-180.

Department of Mathematics, The Johns Hopkins University, Baltimore, Maryland 21218 